\title{
PULMONARY EMPHYSEMA ANALYSIS USING SHEARLET BASED TEXTURES AND RADIAL BASIS FUNCTION NETWORK
}

\author{
Wogderes Semunigus, \\ Department of Electrical and Computer Engineering, \\ Blue Hora University, \\ Blue Hora, Ethiopia, Africa. \\ wogmust@bhu.edu.et/wogmust@gmail.com
}

Submitted: Nov, 30, 2019 Revised: Mar, 12, 2020 Accepted: Mar, 27, 2020

\begin{abstract}
The emergence of High Resolution Computed Tomography (HRCT) images of the lungs clearly shows the parenchymal lung architecture and thus the quantification of obstructive lung disease becomes most accurate. In this study, an automated system to diagnose obstructive lung disease called emphysema is presented using HRCT images of the lungs. The kind of texture information that ideally can be extracted from HRCT images depends on the multi-resolution representation system. The proposed Pulmonary Emphysema Analysis (PEA) system employs Shearlets as it can extracts more texture information than wavelets in different directions and levels. Radial Basis Function Network (RBFN) is employed for the classification of HRCT images into three categories; Normal Tissue (NT), Paraseptal Emphysema (PSE) and Centrilobular Emphysema (CLE). Results prove that a confident diagnosis of pulmonary emphysema is established to help clinicians which will also increase the precision of diagnosis.
\end{abstract}

Keywords: Pulmonary emphysema, shearlets, computed tomography, pulmonary diseases, neural network.

\section{INTRODUCTION}

Today's non invasive medical diagnosis based on images from different imaging modalities is increasingly. The problem of computerized diagnosis system has received considerable interest in the literature where the uses of various techniques are addressed. Local Binary Pattern (LBP) based texture approach is employed in [1] for the quantitative measures of emphysema in HRCT images. It uses the following texture features; intensity histograms, LBP and joint LBP. kNearest Neighbour ( $\mathrm{kNN}$ ) is used as classifier with histogram dissimilarity distance measure.

A texton based approach is discussed in [2] for the classification of emphysema. It has the following stages; codebook generation, learning texton histograms and testing. The former one uses a K-Means Clustering (KMC) algorithm for the construction of codebook and the later stages uses Support Vector Machine (SVM) and kNN for the classification. The same procedure is used in [3] with multiple classifiers to improve the classification accuracy. The parameters such as $k$ in KMC algorithm and texton size are varied for performance evaluation.

Gaussian Markov Random Field (GMRF) is discussed in [4] for emphysema quantification. Before extracting the GMRF parameters, supervised tissue segmentation is employed. The local distributions of spatially varied parameters of GMRF are estimated and represented by normalized histograms as texture 
features. The conventional Gabor filter is extended for emphysema classification in [5]. It uses LBP and various statistical features from the Gabor coefficients at different orientation bands. After extraction, kernel fisher analysis is used for dimension reduction and $\mathrm{kNN}$ is used for the classification.

Convolution Neural Network (CNN) is utilized in [6] for emphysema classification. It has a single unit for feature extraction and recognition of different emphysema patterns. Features are extracted from multi layers of CNN and back propagation learning algorithm is used. Feature ensemble approach is employed for emphysema classification in [7]. It consists of intensity histogram, mean difference technique and log-Gabor filters. These features are concatenated and then reduced by Principal Component Analysis (PCA). Random forest classifier is used as a classifier.

Structural co-occurrence based features are discussed in [8] for emphysema quantification. It analyzes the relationship between two signals in ndimensional space based on the low-level structures. From the relationship, different attributes such as inverse difference moment, entropy, and complementary absolute difference are extracted and SVM classifier is used for the classification. Wavelet transform based emphysema classification is discussed in [9]. After decomposing at 5-levels, histogram is constructed and the feature space is reduced by probabilistic PCA. For classification, kNN, and artificial neural networks are used.

Fractal Dimension (FD) based emphysema classification is described in [10]. The box counting method and holder exponent are applied to calculate the FD of HRCT images. The patterns are detected using ANOVA statistical analysis. Fuzzy decision tree based emphysema classification is discussed in [11]. The local diagonal exterme pattern is used as features and particle swarm optimization is used to partition the feature space. A local diagonal Laplacian pattern is introduced in [12] for emphysema classification. The distance between the histograms of the each pattern is computed and analyzed by ANOVA method. LBP based system is discussed in [13] with the help of SVM classifier for emphysema classification. Three types of LBP are used instead of local LBP and also statistical measurements are used as features.

In this study, an automated system to diagnose pulmonary emphysema is presented with the help of HRCT images. The salient features of the PEA system is the use of Shearlets to extract statistical as well as concurrence descriptors. Also, RBFN is utilized to work with these two sets of features. The organization of this research article is as follows: In section 2, the flow of PEA system along with Shearlet features and RBFN classification are explained in detail. The obtained results and discussions about the performances of PEA system are given in section 3.The last section concludes the proposed classification system.

\section{METHODS AND MATERIALS}

One of the most important factors that affect the results of a screening program is the performance of the clinicians, in interpreting appropriately the visual information presented on the imaging modalities. The probability of observer error is high, due to the incidence of abnormality being very low and the minimum variation of structures between normal and abnormal tissues. To overcome this, PEA system is designed in this work for the classification of lungs tissues from HRCT lung images. Figure 1 shows the overview of PEA system. 


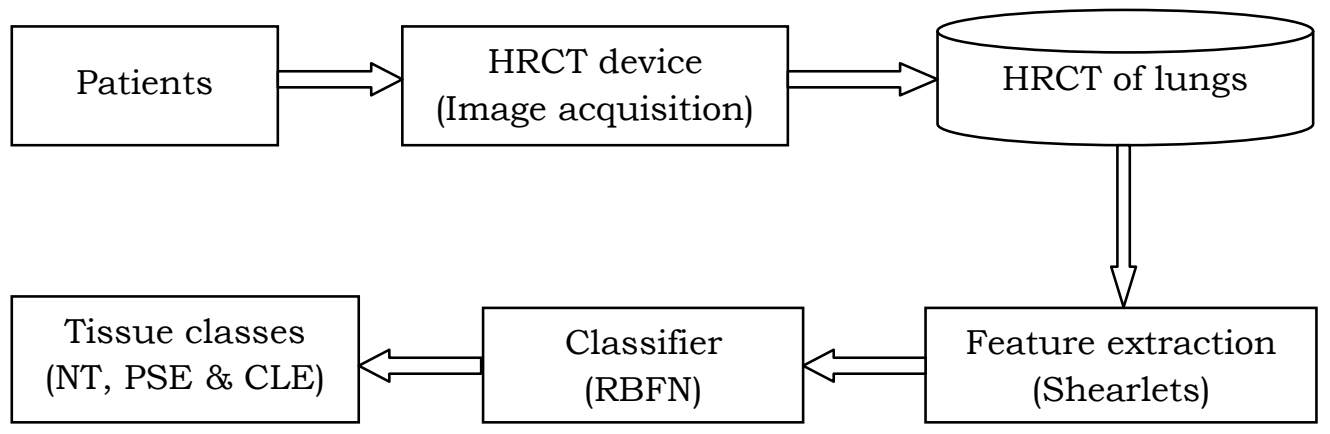

Fig. 1 Overview of PEA system

The proposed PEA system is very similar to the conventional image analysis methods by focusing on feature extraction and classification. The feature extraction method includes Shearlets based image representation algorithm followed by a statistical and concurrence feature extraction from its representation. Then, a pattern recognition technique using RBFN is applied to classify the suspected tissue regions into NT, PSE and CLE. The following sub-section discusses Shearlets based feature extraction and RBFN based classification briefly.

\section{A. Shearlets Based Feature Extraction}

Wavelet transform [14] has the ability to detect pointwise singularities in signals effectively. Due to its isotropic support, it fails to capture the geometric regularity along the surfaces. Several techniques are discussed in [15-16] to exploit the anisotropic regularity of a surface. Among them Shearlets [17-18] provides nearly optimal approximation. The main advantage of affine systems is the ability to combine the geometry and multi-scale analysis. The form of affine system is as follows:

$$
\mathrm{A}_{A B}(\psi)=\left\{\psi_{j, l, k}(x)=|\operatorname{det} A|^{j / 2} \psi\left(B^{l} A^{j} x-k\right): j, l \in \mathfrak{R}, k \in \mathfrak{R}^{2}\right.
$$

where $\psi \in L^{2}\left(\Re^{2}\right), A, B$ are $2 \times 2$ invertible matrices and $|\operatorname{det} B|=1$. If $\sum_{j, l, k}\left|\left\langle f, \psi_{j, l, k}\right\rangle\right|^{2}=\|f\|^{2}$ then $\mathrm{A}_{A B}(\psi)$ forms a Parseval or tight frame. Here the scale transformations are achieved by $A^{j}$ and geometrical transformation by $B^{l}$ to achieve rotations and shear. Like in wavelets, these frames provide not only scales and rotations but also directions. The anisotropic dilation $\left(A=A_{0}\right)$ and shear matrix $\left(B=B_{0}\right)$ for Shearlets are as follows:

$$
\begin{aligned}
& A_{0}=\left(\begin{array}{ll}
4 & 0 \\
0 & 2
\end{array}\right) \\
& B_{0}=\left(\begin{array}{ll}
1 & 1 \\
0 & 1
\end{array}\right)
\end{aligned}
$$

Figure 2 shows the frequency domain induced by Shearlets on the cone. 


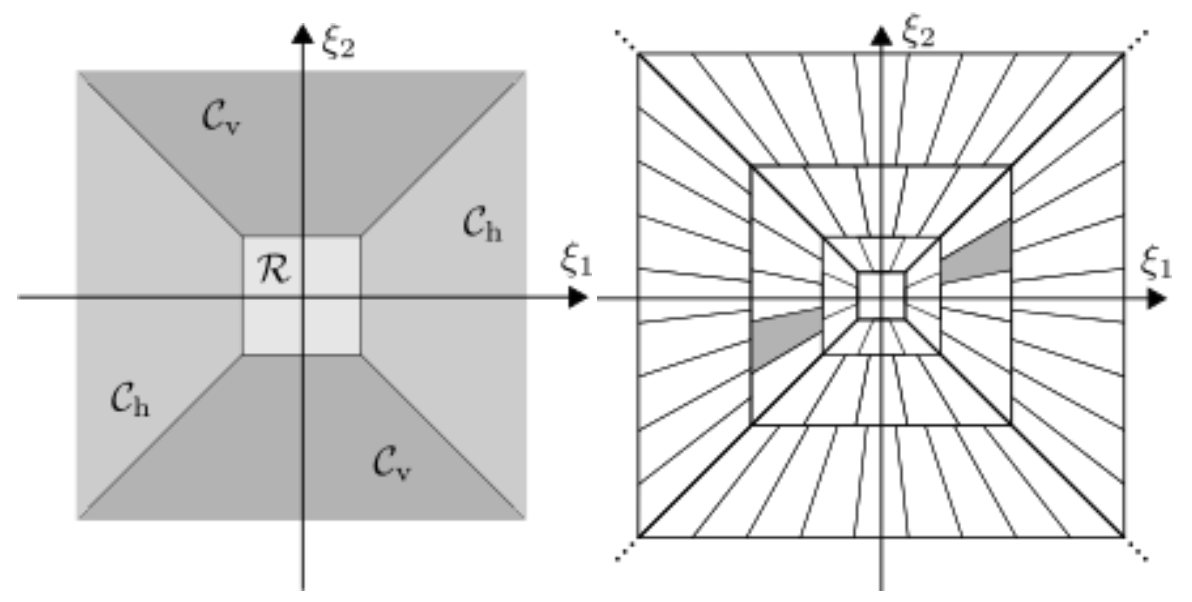

Fig. 2 Frequency domain of Shearlets

The important features used in many medial image analysis systems [1920] which uses are energy and entropy features. Also, the spatial information's are described by the co-occurrence features. These two set of features are combined for tissue classification linearly using HRCT lung image. Table 1 shows the extracted features.

TABLE 1 Description of features

\begin{tabular}{|c|c|}
\hline Features & Description \\
\hline \multicolumn{2}{|c|}{ Statistical features } \\
\hline Entropy & $\frac{1}{M N} \sum_{m=1}^{M} \sum_{n=1}^{N} S S(m, n)$ \\
\hline Energy & $-\sum_{n} p_{n} \log _{2}\left(p_{n}\right)$ \\
\hline \multicolumn{2}{|c|}{ co-occurrence features } \\
\hline Energy & $\sum_{m, n} c o(m, n)^{2}$ \\
\hline Contrast & $\sum_{m, n}|m-n|^{2} \operatorname{co}(m, n)^{2}$ \\
\hline Homogeneity & $\sum_{m, n} \frac{c o(m, n)}{1+|m-n|}$ \\
\hline \multicolumn{2}{|c|}{$\begin{array}{c}S S \text { represents the Shearlets sub-band of size } M \times N \\
\text { co is the normalized co-occurrence matrix of } S S . . \\
S S \text { coordinates are represented by }(m, n) . \\
P n \text { is the probability with gray level } n\end{array}$} \\
\hline
\end{tabular}

A total of 5 features described in table 1 are extracted from each subbands after representation by Shearlets filters. As it provides more directional sub-bands than wavelets, more features are extracted at each level that depends on the number of directions. Thus, more discriminating features can be extracted by using Shearlets based representation system. These features are then classified using RBFN. 


\section{B. Classification using RBFN}

The sets of features obtained from the previous step are used to build RBFN classifier [21]. The RBFN classifier is a kind of neural network with a structure similar to conventional neural networks built to estimate the conditional probability density functions, $P\left(x \mid w_{i}\right)$, for each class $w_{i}$. Each density is estimated as a finite mixture of uncorrelated Gaussians $G\left(x ; \mu_{j}, \sigma_{j}\right)$ centered at $\mu_{j}$ and with a diagonal covariance matrix of components $\sigma_{j}$

$$
P(x \mid w)=\sum_{j=1}^{M} W_{j} G\left(x ; \mu_{j}, \sigma_{j}\right)
$$

where $W_{j}$ is the weight associated with the $j^{\text {th }}$ component of the mixture. The network is then trained using the log-likelihood as an optimality criterion. Such a network is shown in Figure 3.

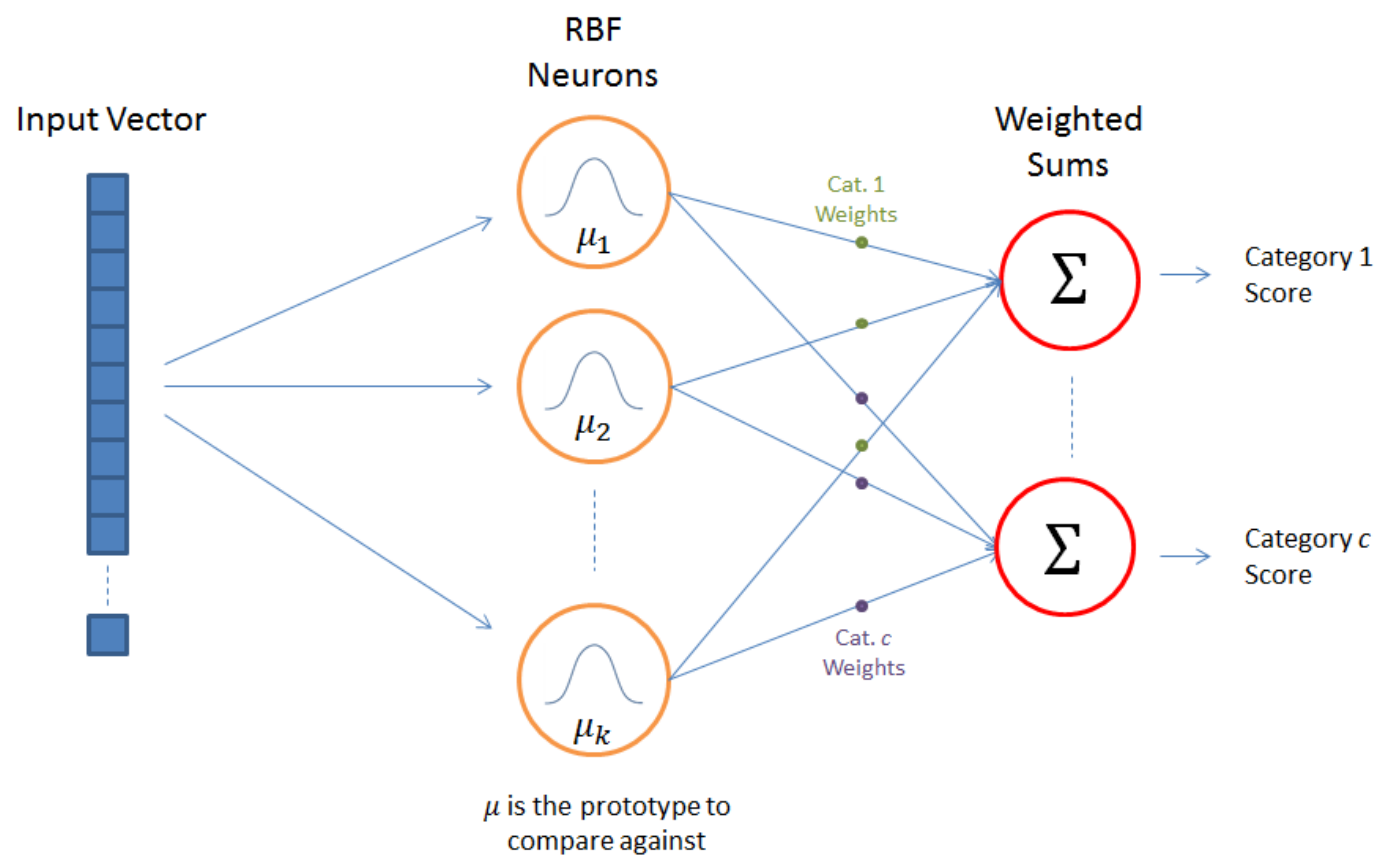

Fig. 3 Block diagram of RBFN

The number $M$ of hidden neurons of the power density function approximating network is selected using a method referred to as Maximum Penalised Likelihood (MPL).

$$
\begin{aligned}
& \text { MPL }=\log -\text { likelihood }+ \text { Penalty } \\
& \text { Penalty }=-\frac{d p \log N}{2 N}
\end{aligned}
$$

where $p$ is the total number of parameters to be estimated, $d$ is the data dimensionality and $N$ is the number of training samples. 
Once the densities, $P\left(x \mid w_{i}\right)$ have been estimated, the classifier is built through the usual Bayes rule. For the two class problem, $w=1,2$, the classifier is built as shown in Figure 4.

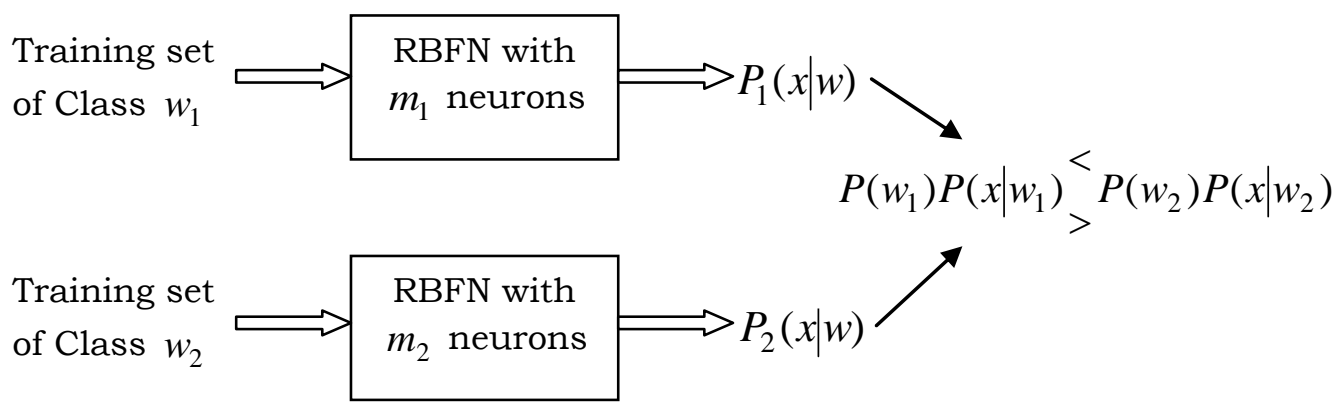

Fig. 4 Bayes decision rules

Having an estimate of the power density functions not only defines a decision boundary between the two classes, but it also gives an insight about the data structure. It allows us to identify the presence of outliers: points that should neither be classified as normal, nor as abnormal, because there is little evidence that they come from either class. In order to detect possible outliers, two thresholds have been defined;

$$
\begin{aligned}
& T_{w 1}=\min _{x \in X_{w 1}} P\left(x \mid w_{1}\right) \\
& T_{w 2}=\min _{x \in X_{w 2}} P\left(x \mid w_{2}\right)
\end{aligned}
$$

Where $X_{w 2}$ and $X_{w 2}$ are the training sets for the two classes. The definitions for outliers are given below:

$$
P\left(x \mid w_{1}\right)<T_{w 1} \text { and } P\left(x \mid w_{2}\right)<T_{w 2}
$$

\section{RESULTS AND DISCUSSION}

The performance of PEA system is evacuated using freely available HRCT images from Computed Tomography Emphysema Database (CTED) [1] images. These images are acquired using general electric equipment. CTED consists of 115 HRCT slices of upper, middle and lower part of lungs of 39 subjects. Among 39 subjects, 30 are smokers and 9 are never smokers. These images are assessed by a CT pulmonologist and a chest radiologist and the leading patterns such as NT, PSE and CLE are marked as ground truth data. A total of 168 patches with a resolution of $61 \times 61$ pixels are readily available in CTED for performance evaluation. Table 2 gives the details of CTED briefly and Figure 5 shows sample images in CTED containing NT, PSE and CLE patterns.

TABLE 2 Description of CTED

\begin{tabular}{|c|c|c|c|c|}
\hline \multirow{2}{*}{ \#total images } & \multirow{2}{*}{$\begin{array}{c}\text { \#patch resolution } \\
\text { (pixels) }\end{array}$} & \multicolumn{3}{|c|}{ Observations } \\
\cline { 3 - 5 } & \#PSE & \#CLE & \#NT \\
\hline 168 & $61 \times 61$ & 59 & 50 & 59 \\
\hline
\end{tabular}




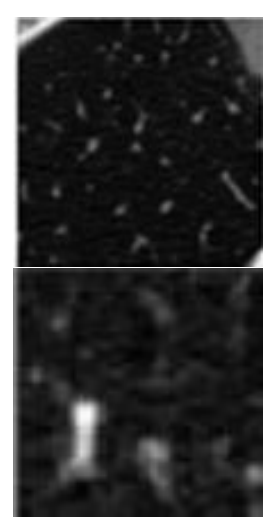

(a)

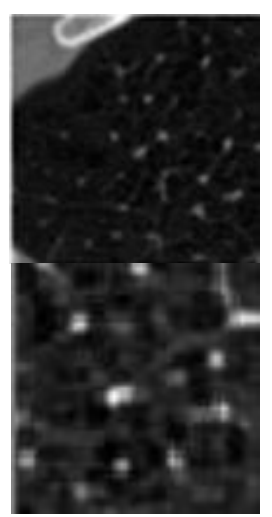

(b)

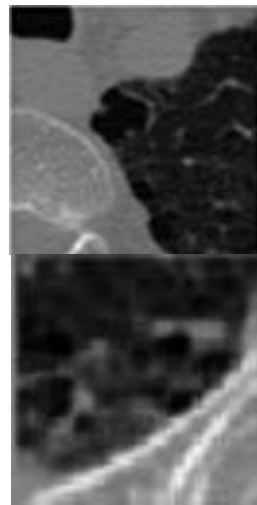

(c)

Fig. 5 Sample image in CTED (a) NT (b) CLE (c) PSE

The RBFN classifier is evaluated using Leave-One-Out (LOO) error estimation on the HRCT images in the CTED. In the LOO method, one sample is excluded from the training set and the classifier is designed using the remaining $\mathrm{N}-1$ samples. The excluded sample is then used for testing the RBFN classifier. This operation is repeated $N$ times to test all the training samples. The number of misclassified samples is counted to obtain an estimate of the error. Since each test image measurement is excluded from the design sample set, the independence between the design and test sets is maintained. The number of correctly classified samples is then counted to obtain an estimate of the RBFN performance. Based on the outputs, confusion matrices are drawn.

TABLE 3 CM and performance metrics for 1-Level Shearlets features

\begin{tabular}{|c|c|c|c|c|c|c|c|}
\hline $\begin{array}{l}\text { Shearlets } \\
\text { Directions }\end{array}$ & Pattern & \#PSE & \#CLE & \#NT & Accuracy & Sensitivity & Specificity \\
\hline \multirow{3}{*}{2} & \#PSE & 44 & 9 & 6 & 80.95 & 74.58 & 84.40 \\
\hline & \#CLE & 9 & 34 & 7 & 80.95 & 68.00 & 86.44 \\
\hline & \#NT & 8 & 7 & 44 & 83.33 & 74.58 & 88.07 \\
\hline \multicolumn{5}{|c|}{ Average } & 81.75 & 72.38 & 86.31 \\
\hline \multirow{3}{*}{4} & \#PSE & 48 & 7 & 4 & 85.71 & 81.36 & 88.07 \\
\hline & \#CLE & 6 & 39 & 5 & 86.90 & 78.00 & 90.68 \\
\hline & \#NT & 7 & 4 & 48 & 88.10 & 81.36 & 91.74 \\
\hline \multicolumn{5}{|c|}{ Average } & 86.90 & 80.24 & 90.16 \\
\hline \multirow{3}{*}{8} & \#PSE & 51 & 3 & 5 & 89.88 & 86.44 & 91.74 \\
\hline & \#CLE & 4 & 42 & 4 & 91.67 & 84.00 & 94.92 \\
\hline & \#NT & 5 & 3 & 51 & 89.88 & 86.44 & 91.74 \\
\hline \multicolumn{5}{|c|}{ Average } & 90.48 & 85.63 & 92.80 \\
\hline \multirow{3}{*}{16} & \#PSE & 48 & 4 & 7 & 87.95 & 81.36 & 91.59 \\
\hline & \#CLE & 3 & 40 & 4 & 90.36 & 85.11 & 92.44 \\
\hline & \#NT & 6 & 5 & 49 & 86.75 & 81.67 & 89.62 \\
\hline \multicolumn{5}{|c|}{ Average } & 88.35 & 82.71 & 91.22 \\
\hline
\end{tabular}


TABLE 4 CM and performance metrics for 2-Level Shearlets features

\begin{tabular}{|c|c|c|c|c|c|c|c|}
\hline $\begin{array}{l}\text { Shearlets } \\
\text { Directions }\end{array}$ & Pattern & \#PSE & \#CLE & \#NT & Accuracy & Sensitivity & Specificity \\
\hline \multirow[t]{3}{*}{ 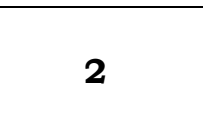 } & \#PSE & 46 & 8 & 5 & 83.33 & 77.97 & 86.24 \\
\hline & \#CLE & 8 & 36 & 6 & 83.33 & 72.00 & 88.14 \\
\hline & \#NT & 7 & 6 & 46 & 85.71 & 77.97 & 89.91 \\
\hline \multicolumn{5}{|c|}{ Average } & 84.13 & 75.98 & 88.09 \\
\hline \multirow{3}{*}{4} & \#PSE & 50 & 7 & 2 & 86.90 & 84.75 & 88.07 \\
\hline & \#CLE & 6 & 41 & 3 & 89.29 & 82.00 & 92.37 \\
\hline & \#NT & 7 & 2 & 50 & 91.67 & 84.75 & 95.41 \\
\hline \multicolumn{5}{|c|}{ Average } & 89.29 & 83.83 & 91.95 \\
\hline \multirow{3}{*}{8} & \#PSE & 53 & 3 & 3 & 93.45 & 89.83 & 95.41 \\
\hline & \#CLE & 2 & 44 & 4 & 92.86 & 88.00 & 94.92 \\
\hline & \#NT & 3 & 3 & 53 & 92.26 & 89.83 & 93.58 \\
\hline \multicolumn{5}{|c|}{ Average } & 92.86 & 89.22 & 94.64 \\
\hline \multirow{3}{*}{16} & \#PSE & 50 & 4 & 5 & 89.76 & 84.75 & 92.52 \\
\hline & \#CLE & 3 & 42 & 2 & 92.17 & 89.36 & 93.28 \\
\hline & \#NT & 5 & 4 & 51 & 90.36 & 85.00 & 93.40 \\
\hline \multicolumn{5}{|c|}{ Average } & 90.76 & 86.37 & 93.07 \\
\hline
\end{tabular}

TABLE 5 CM and performance metrics for 3-Level Shearlets features

\begin{tabular}{|c|c|c|c|c|c|c|c|}
\hline $\begin{array}{l}\text { Shearlets } \\
\text { Directions }\end{array}$ & Pattern & \#PSE & \#CLE & \#NT & Accuracy & Sensitivity & Specificity \\
\hline \multirow{3}{*}{2} & \#PSE & 50 & 6 & 3 & 88.10 & 84.75 & 89.91 \\
\hline & \#CLE & 6 & 40 & 4 & 88.10 & 80.00 & 91.53 \\
\hline & \#NT & 5 & 4 & 50 & 90.48 & 84.75 & 93.58 \\
\hline \multicolumn{5}{|c|}{ Average } & 88.89 & 83.16 & 91.67 \\
\hline \multirow{3}{*}{4} & \#PSE & 54 & 4 & 1 & 91.67 & 91.53 & 91.74 \\
\hline & \#CLE & 4 & 44 & 2 & 94.05 & 88.00 & 96.61 \\
\hline & \#NT & 5 & 0 & 54 & 95.24 & 91.53 & 97.25 \\
\hline \multicolumn{5}{|c|}{ Average } & 93.65 & 90.35 & 95.20 \\
\hline \multirow{3}{*}{8} & \#PSE & 58 & 1 & 0 & 98.81 & 98.31 & 99.08 \\
\hline & \#CLE & 1 & 49 & 0 & 98.81 & 98.00 & 99.15 \\
\hline & \#NT & 0 & 0 & 59 & 100.00 & 100.00 & 100.00 \\
\hline \multicolumn{5}{|c|}{ Average } & 99.21 & 98.77 & 99.41 \\
\hline \multirow{3}{*}{16} & \#PSE & 56 & 2 & 1 & 94.67 & 94.92 & 94.55 \\
\hline & \#CLE & 3 & 46 & 1 & 95.27 & 92.00 & 96.64 \\
\hline & \#NT & 3 & 2 & 55 & 95.86 & 91.67 & 98.17 \\
\hline \multicolumn{5}{|c|}{ Average } & 95.27 & 92.86 & 96.45 \\
\hline
\end{tabular}


TABLE 6 CM and performance metrics for 4-Level Shearlets features

\begin{tabular}{|c|c|c|c|c|c|c|c|}
\hline $\begin{array}{l}\text { Shearlets } \\
\text { Directions }\end{array}$ & Pattern & \#PSE & \#CLE & \#NT & Accuracy & Sensitivity & Specificity \\
\hline \multirow{3}{*}{2} & \#PSE & 48 & 7 & 4 & 85.71 & 81.36 & 88.07 \\
\hline & \#CLE & 7 & 38 & 5 & 85.71 & 76.00 & 89.83 \\
\hline & \#NT & 6 & 5 & 48 & 88.10 & 81.36 & 91.74 \\
\hline \multicolumn{5}{|c|}{ Average } & 86.51 & 79.57 & 89.88 \\
\hline \multirow{3}{*}{4} & \#PSE & 52 & 5 & 2 & 89.88 & 88.14 & 90.83 \\
\hline & \#CLE & 4 & 43 & 3 & 92.26 & 86.00 & 94.92 \\
\hline & \#NT & 6 & 1 & 52 & 92.86 & 88.14 & 95.41 \\
\hline \multicolumn{5}{|c|}{ Average } & 91.67 & 87.42 & 93.72 \\
\hline \multirow{3}{*}{8} & \#PSE & 55 & 1 & 3 & 95.24 & 93.22 & 96.33 \\
\hline & \#CLE & 2 & 46 & 2 & 95.83 & 92.00 & 97.46 \\
\hline & \#NT & 2 & 2 & 55 & 94.64 & 93.22 & 95.41 \\
\hline \multicolumn{5}{|c|}{ Average } & 95.24 & 92.81 & 96.40 \\
\hline \multirow{3}{*}{16} & \#PSE & 52 & 2 & 5 & 92.77 & 88.14 & 95.33 \\
\hline & \#CLE & 1 & 44 & 2 & 95.18 & 93.62 & 95.80 \\
\hline & \#NT & 4 & 3 & 53 & 91.57 & 88.33 & 93.40 \\
\hline \multicolumn{5}{|c|}{ Average } & 93.17 & 90.03 & 94.84 \\
\hline
\end{tabular}

It is evident from Tables 3 to 6 , the features from Shearlets provides 99.21\% accuracy for emphysema classification when the features are from 8 directions of $3^{\text {rd }}$ level. To visually analyze the performance of PEA system, the maximum results obtained from each Shearlets level are plotted in Figure 6.

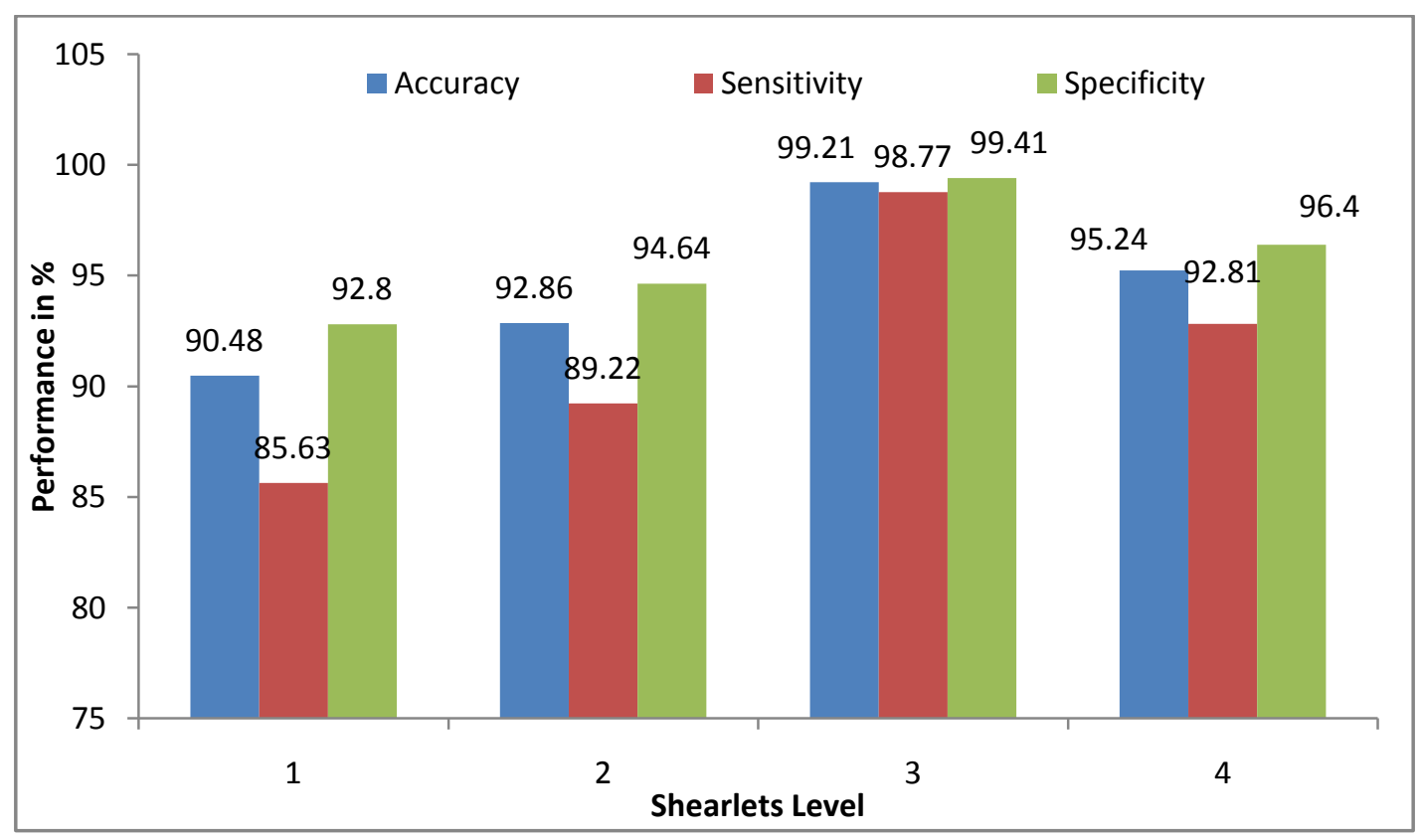

Fig. 6 Maximum performance of PEA system at each level

From the results, the PEA system acts as a second reader, resulting in a reduction of the number of expert radiologists where double reading is the current 
practice. The system has the ability to prompt three kinds of abnormalities and robust to provide the second opinion for an expert radiologist in analyzing HRCT lung images for pulmonary emphysema.

\section{CONCLUSION}

In this work, HRCT medical image interpretation is considered and the method of Shearlets and RBFN are employed as a possible solution for the classification of pulmonary emphysema. The extended directional decomposition of the lungs is obtained using Shearlets. From the Shearlet representation with various levels and directions two sets of features; statistical and co-occurrence features are extracted. The LOO error estimation is used to analyze the performance of RBFN classifier with 168 images. Results confirm the reliability of the classification system to classify the HRCT images into three classes: NT, PSE and CLE with an average accuracy of $99.21 \%$. In addition, the structural information in various directional sub-bands improves the discriminating power of the RBFN classifier. The PEA system performs well as an experienced radiologist that improve the observer's performance and in such a case, the necessity for double reading will be reduced.

\section{REFERENCES}

[1]. L. Sørensen, S.B. Shaker, and M. de Bruijne, "Quantitative Analysis of Pulmonary Emphysema using Local Binary Patterns, IEEE Transactions on Medical Imaging, Vol. 29, No. 2, 2010, pp. 559-569.

[2]. M.J. Gangeh, L. Sørensen, S.B. Shaker, M.S. Kamel, M. de Bruijne, and M. Loog, "A texton-based approach for the classification of lung parenchyma in CT images", International conference on Medical Image Computing and Computer Assisted Intervention, 2010, pp. 595-602.

[3]. M.J. Gangeh, L. Sørensen, S.B. Shaker, M.S. Kamel and M. de Bruijne, "Multiple classifier systems in texton-based approach for the classification of CT images of lung", International workshop on Medical Computer Vision . Recognition Techniques and Applications in Medical Imaging, 2010, pp. 153-163.

[4]. C. Dharmagunawardhana, S. Mahmood, M. Bennett, and M. Niranjan, "Quantitative analysis of pulmonary emphysema using isotropic Gaussian Markov random fields", International Conference on Computer Vision Theory and Applications, 2014, pp. 44-53

[5]. R. Nava, B. Escalante-Ramírez, G. Cristóbal, and R.S.J. Estépar, "Extended Gabor approach applied to classification of emphysematous patterns in computed tomography", Medical \& Biological Engineering \& Computing, Vol. 52, No. 4, 2014, pp. 393-403.

[6]. X. Pei, "Emphysema Classification Using Convolutional Neural Networks", Intelligent Robotics and Applications, 2015, pp. 455-461.

[7]. R. Nava, J. Olveres, J. Kybic, B. Escalante, G. Cristóbal, "Feature Ensemble for Quantitative Analysis of Emphysema in CT imaging", IEEE International Conference on E-Health and Bioengineering, 2015, pp. 1-4.

[8]. G.L.B. Ramalhoa, D.S. Ferreiraa, P.P.R. Filhoa, F.N.S. de Medeiros, "Rotation-invariant Feature Extraction using a Structural Co-occurrence Matrix", Measurement, Vol. 94, 2016, pp. 406-415. 
[9]. V. Srivastava and R.K. Purwar, "A Five-Level Wavelet Decomposition and Dimensional Reduction Approach for Feature Extraction and Classification of MR and CT Scan Images", Applied Computational Intelligence and Soft Computing, Vol. 2017, 2017, pp. 1-9.

[10]. M.A. Ibrahim, O.A. Ojo, P.A. Oluwafisoye, "Identification of emphysema patterns in high resolution computed tomography images", Journal of Biomedical Engineering and Informatics, Vol. 4, No. 1, 2018, pp. 16-24.

[11]. S.J. Narayanan, R. Soundrapandiyan, B. Perumal, C.J. Baby, "Emphysema Medical Image Classification Using Fuzzy Decision Tree with Fuzzy Particle Swarm Optimization Clustering", International Conference on Smart Intelligent Computing and Applications, 2018, pp. 305-313.

[12]. P.K.R. Yelampalli, J. Nayak, V.H. Gaidhane, "A novel binary feature descriptor to discriminate normal and abnormal chest $\mathrm{CT}$ images using dissimilarity measures", Pattern Analysis and Applications, Vol. 22, No. 4, 2019, pp. 1517-1526.

[13]. E. Tuba, I. Strumberger, N. Bacanin, M. Tuba, "Analysis of local binary pattern for emphysema classification in lung CT image", IEEE International Conference on Electronics, Computers and Artificial Intelligence, 2019, pp. 1-5 .

[14]. Mallat S. A wavelet tour of signal processing: the sparse way. Academic Press; 2008

[15]. M.N. Do and M. Vetterli, "The contourlet transform: an efficient directional multiresolution image representation", IEEE Transactions on image processing, Vol. 14, no. 12, 2005, pp. 2091-2106.

[16]. D. Donoho and E. Candes, "Continuous curvelet transform: II. Discretization and frames", Applied and Computational Harmonic Analysis, Vol. 19, No. 2, 2005, pp. 198-222.

[17]. W.Q. Lim, "The discrete shearlet transform: a new directional transform and compactly supported shearlet frames", IEEE Transactions on Image Processing, Vol. 19, No. 5, 2010, pp. 1166-1180.

[18]. G. Easley, D. Labate and W.Q. Lim, "Sparse directional image representations using the discrete shearlet transform", Applied and Computational Harmonic Analysis, Vol. 25, No. 1, 2008, pp. 25-46.

[19]. R.R. Ayalapogu, S. Pabboju and R.R. Ramisetty, "Analysis of dual tree Mband wavelet transform based features for brain image classification", Magnetic resonance in medicine, Vol. 80, no. 6, 2018, pp. 2393-2401.

[20]. K.S. Thivya, P. Sakthivel and P.M. Venkata Sai, "Analysis of framelets for breast cancer diagnosis", Technology and Health Care, Vol. 24, No. 1, 2016, pp. 21-29.

[21]. L. Sardo and J. Kittler, "Minimum complexity estimator for rbf networks architecture selection", International Conference on Neural Networks, Vol. 1, 1996, pp. 137-142. 